\title{
La conservation des ressources phyto-génétiques en réseau : incitations économiques et contraintes institutionnelles
}

\author{
Michel TrommetTer \\ Inra-SERD, Université Pierre Mendès-France, BP 47, \\ 38040 Grenoble Cedex 9, France
}

Résumé - Dans cet article, notre objectif est de définir, pour les acteurs publics et privés, le fonctionnement institutionnel et le système d'incitation facilitant leur adhésion à la charte de conservation des ressources phytogénétiques. Différentes configurations de réseau et d'incitations seront analysées via les règles internes aux réseaux : répartition des coûts, accès, responsabilité, durée de l'engagement, etc. Ces configurations doivent intégrer le niveau international (relations bilatérales, multilatérales), les différentes formes de droits de propriété intellectuelle (nationales et internationales).

économie / conservation / ressources génétiques / réseaux de conservation / recherche coopérative / recherche \& développement

\begin{abstract}
Networks for the conservation of plant genetic resources: economic incentives and institutional constraints. In this article, our objective is to define, for private and public actors, the institutional mechanisms and the incentive systems facilitating their participation to the French "conservation charter" for plant genetic resources. Different system and incentive configurations will be analyzed via internal rules of the systems: distribution of costs, access, responsibility, commitment duration, etc. These configurations have to integrate the international level (bilateral relationships, multilateral) and the different form of intellectual property rights (national and international.
\end{abstract}

economy / conservation / genetic resource / conservation network / cooperation / research and development

* Correspondance et tirés-à-part

E-mail: trommetter@grenoble.inra.fr 


\section{INTRODUCTION}

Devant l'importance du secteur agricole et agro-alimentaire en France (particulièrement de la filière semence), on trouve de nombreuses collections ex-situ de ressources génétiques tant dans les instituts publics de recherche que dans les laboratoires privés ${ }^{1}$. Dans ce contexte et si nous étudions ces collections dans un objectif de conservation de la diversité biologique, nous pouvons identifier des limites à cette organisation : une perte du matérie. considéré comme non stratégique à court terme ; un niveau de redondance élevé. En effet, les collections de laboratoire (fonction identique à celle des collections des sélectionneurs) sont tributaires des programmes de recherche. Face à ces difficultés, au début des années 1990, le Bureau des ressources génétiques (BRG) a proposé d'organiser, en coordination avec l'ensemble des acteurs (publics et privés), ces collections dans un objectif de conservation « en réseau », en particulier avec la création de Collections Nationales $(\mathrm{CN})$ qui visent à préserver, par espèce, la diversité biologique pour un usage futur parallèlement aux collections de travail (de court terme). Ces $\mathrm{CN}$, contrairement à ce qui se passe dans de nombreux pays (Norvège, Allemagne, Pays Bas, etc.), ne sont pas centralisées géographiquement mais sont éclatées dans différents laboratoires publics et privés avec pour chaque espèce un responsable de réseau. C'est pourquoi nous allons, dans un premier temps, présenter les différentes phases de la conservation des ressources génétiques et de leur organisation en France, après quoi nous étudierons les mécanismes incitatifs qui ont conduit des acteurs publics et privés à participer à un tel programme. Notre analyse est basée sur l'étude de plusieurs réseaux parmi la trentaine qui sont en train de se mettre en place actuellement et qui intègrent des acteurs publics et privés ${ }^{2}$.

\section{LA PROBLÉMATIQUE DE LA CONSERVATION EN RÉSEAU}

Généralement, les banques de génotypes ont pour mission de conserver du matériel plus ou moins primitif véhiculant des gènes utiles en sélection. Dans ce contexte, nous allons étudier comment la conservation en réseau a pu se développer en France et nous en présenterons les spécificités organisationnelles. Enfin, nous verrons les enjeux liés à la mise en œuvre de cette organisation au niveau national, mais également en rapport avec les négociations internationales (Convention sur la diversité biologique, CDB, Organisation mondiale du commerce, OMC, etc.).

\footnotetext{
${ }^{1}$ Il existe également une conservation mise en place pour des raisons éthiques, dont la justification est généralement basée sur l'évaluation du consentement à payer de la société, évaluation réalisée par la « méthode d'évaluation contingente » [3].

${ }^{2}$ Notre étude est basée sur l'analyse de 5 réseaux selon : des entretiens directs dans le cadre des réseaux maïs et céréales à paille ; des documents et articles disponibles dans le cadre des réseaux tournesol, betteraves et prunus.
} 


\subsection{Un contexte et des objectifs particuliers}

Dans certains cas, le réseau de conservation des ressources génétiques s'appuie sur une structure préexistante sous la forme d'une association (professionnelle) ou de groupement d'intérêt économique, GIE, (l'association des producteurs de maïs, Promaïs, l'association des établissements obtenteurs et producteurs de semences oléagineuses, Amsol, etc.). Ces entités avaient et ont encore un rôle dans d'autres volets que la conservation des ressources génétiques, en parti culier dans le cas de contrats de recherches (multi-partenaires publics et privés, tel le « programme population source » chez le maïs) liées à la mise au point de nouveaux produits ou de programmes de sélection à faible intensité compétitive (mise au point de matériel pré-compétitif).

\subsubsection{Les objectifs stratégiques de la conservation}

L'intérêt économique et social de la conservation des ressources génétiques est plus ou moins bien reconnu par la collectivité. Les objectifs de la conservation peuvent être abordés sous deux angles : la précaution et le progrès génétique.

L'analyse de la conservation des ressources génétiques par une approche « principe de précaution » a son origine dans l'étude des risques liés au rétrécissement de la base génétique utilisée dans les variétés commerciales ce qui peut avoir des conséquences économiques et sociales irréversibles en cas d'apparition d'un pathogène ${ }^{3}$. Dans ce contexte, nous pouvons montrer que l'avantage de la conservation sur la non-conservation peut justifier les dépenses pour créer des collections et ce, même pour des probabilités faibles d'apparition d'un pathogène $[14,26]$.

$\mathrm{Au}$ niveau du progrès génétique, il existe pour la plupart des grandes espèces, mais il est également reconnu que la base génétique du matériel utilisé reste étroite. Dans ce cadre, une question à résoudre est de savoir si le gain social lié à un meilleur progrès technique justifie les dépenses en recherche pour conserver et exploiter la variabilité génétique [19].

\subsubsection{Le «Réseau », la conservation et la recherche- développement}

Le réseau se charge de la gestion des collections nationales, de la tenue à jour de la base de données, de la coordination de réseaux coopératifs d'observation (évaluation et échange de matériel entre les acteurs). Ainsi, la conservation en réseau a trois objectifs :

- maintenir la collection à long terme (conservation et régénération) ;

\footnotetext{
${ }^{3}$ Les exemples sont très explicites (le maïs aux État-Unis dans les années 1970, ou la pomme de terre en Irlande au XIXe siècle), mais insuffisants pour évaluer le risque actuel et justifier ainsi une approche purement publique de la conservation.
} 
- caractériser la collection afin de limiter les redondances et d'accélérer les potentialités d'utilisation ;

- inciter à la participation par l'évaluation de la collection pour certains caractères d'intérêt agronomique (fonctions).

La stratégie réseau de conservation conduit à impliquer les utilisateurs potentiels dans la conservation. Cette approche permet d'une part de faciliter la coopération entre les entreprises et de conduire à des économies d'échelle (statiques et dynamiques) à la fois sur la conservation et sur les dépenses de Recherche-développement $(\mathrm{R} \& \mathrm{D})$ sur les ressources génétiques et d'autre part d'augmenter la probabilité qu'un programme de R\&D utilisant les ressources génétiques de la collection soit lancé (valorisation de la collection). Parallèlement et pour le sélectionneur, un troisième effet doit être pris en compte : l'augmentation de la probabilité de succès d'un programme. En effet, comme le matériel a été mieux évalué, l'acteur choisit plus facilement les populations sur lesquelles travailler (gain de temps et choix plus large).

\subsection{Les dispositifs de coordination}

Dans le cadre des chartes signées au niveau de chaque réseau, qui est le niveau le plus élevé dans l'harmonisation de l'organisation des collections de ressources génétiques nous pouvons noter quelques points déterminants $[7,17,18,20]$ : l'engagement de chaque partenaire correspond à une participation d'une durée illimitée (représentée par un contrat de 5 ans renouvelable automatiquement) mais avec possibilité de sortie en cas de problème (prenant en compte un délai de réaction pour la cellule coordinatrice) ; la définition de l'organisation du réseau avec la création de comités de coordination.

\subsubsection{Les comités de coordination}

Au niveau de la coordination et du contrôle du bon fonctionnement du réseau il y a deux entités :

- La cellule de coordination qui a un double objectif, d'une part d'animation et d'organisation du réseau avec l'enrichissement et la rationalisation de la CN (étude des demandes d'entrées, de sorties, etc.), et d'autre part la gestion du double de sécurité avec la centralisation des demandes et de la distribution, et la gestion de la base de données. Il s'agit de définir des méthodes de gestion de la variabilité génétique (information et apprentissage).

- Le comité de pilotage a pour rôle de définir les grandes lignes d'activités du réseau. Il est composé d'acteurs du réseau, de représentants de ministères et d'acteurs de la filière. Les rôles de ce comité sont multiples : définir la politique générale et le statut des collections, le règlement intérieur (partage des tâches, conditions de participation, etc.), des conditions d'échange de matériel (local ou étranger), etc. [7]. 


\subsubsection{L'effort demandé aux conservateurs/évaluateurs}

Comme nous venons de le voir, l'organisation interne des réseaux est définie au niveau du comité de pilotage, pour les opérations de gestion de la CN (collecte, caractérisation et évaluation) et de maintien des échantillons de la CN. Néanmoins, compte tenu de l'originalité du mode de conservation en réseau, nous disposons encore d'assez peu de données sur l'effort de chaque acteur pour participer au réseau de conservation, puisqu'il varie selon le règlement intérieur qui n'est pas complètement harmonisé au niveau national - les engagements liés à la signature de la charte permettent donc une relative flexibilité dans la gestion des différents réseaux. Ainsi, deux types d'organisation, particulièrement intéressants, ont pu être recensés :

- Réseau de type 1 (R1) : L'organisation est flexible, chaque participant au réseau est responsable du matériel qu'il met dans la collection tant pour les opérations de conservation que de régénération, évaluation phénotypique et génétique, etc., dans ce contexte généralement la collection de réseau et la $\mathrm{CN}$ seront peu différentiées.

- Réseau de type 2 (R2) : L'organisation est réglementée avec par exemple : la répartition égalitaire des coûts afférents à la conservation et à l'évaluation des collections, soit $50 \%$ pour les acteurs publics et $50 \%$ privés pour les investissements et chaque acteur public ou privé doit financer ou réaliser les tâches liées au fonctionnement du réseau (conservation, évaluation, etc.) pour un même montant. Dans ce cadre les collections de réseaux et les $\mathrm{CN}$ pourront être assez différentes du fait de recherches pré-compétitives.

Les étapes directement liées à la conservation durable des ressources génétiques sont les opérations de conservation, de régénération et de diffusion du matériel. Pour les activités d'évaluation, si la caractérisation primaire est généralement réalisée quel que soit le type de réseau ( $\mathrm{R} 1$ ou $\mathrm{R} 2)$ - via des analyses phénotypiques, et/ou sur la base d'évaluation moléculaire, ces informations étant gérées dans une base de données commune à tous les acteurs du réseau, la caractérisation secondaire et la recherche de « fonctions » dépendent des réseaux concernés et de l'organisation de la recherche (principalement dans le cadre des $\mathrm{R} 2$ ).

Ces étapes d'évaluation et de caractérisation sont nécessaires, car généralement, même si le partage des coûts et l'accès à un plus grand nombre d'échantillons sont cités, les phases de recherche collective de fonctions (en particulier de résistances) et de pré-sélection de matériel (jusqu'à la création de pools géniques) sont mises en avant comme un motif d'adhésion au réseau. En effet, les ressources conservées sont, en partie, constituée d'espèces locales ou de races locales anciennes qui sont plus ou moins éloignées génétiquement d'un cultivar issu d'une sélection poussée et donc difficiles à intégrer en l'état dans des programmes de sélection. Dans ce contexte, une valorisation de ce matériel génétique passe par un travail de présélection d'autant moins coûteux que le nombre de partenaires est élevé et que l'information disponible sur ce 
type de matériel est importante. Dès que des lignées suffisamment améliorées deviennent disponibles, les sélectionneurs se montrent plus intéressés qu'ils ne l'étaient par le matériel brut proposé.

Cette organisation de la conservation en réseau se retrouve quelquefois dans des centres de conservation centralisés où l'on peut rencontrer des activités de recherche coopératives avec des entreprises privées (voir le latin american maize project du Cimmyt, [23]), mais ce n'est pas la généralité.

\subsubsection{Les droits et obligations des conservateurs}

Si nous résumons l'effort demandé aux membres du réseau de conservation, notons qu'ils doivent assurer la conservation et la gestion du matériel qu'ils ont confié au réseau (R1) et éventuellement de celui dont le réseau leur a donné la charge (réseau R2). Par contre, ils n'ont aucun droit physique sur les ressources de la CN, même sur celles qu'ils détiennent. De plus, ils doivent informer le coordonateur des informations dont ils disposent sur le matériel : données de passeport et description primaire figurant dans la base de données $\mathrm{CN}$; alors que des données complémentaires peuvent figurer dans la base de données mais avec un accès réduit (aux membres du réseau), voire avec des effets retards (de 3 à 5 ans avant de diffuser l'information à l'ensemble du réseau selon les réseaux étudiés).

\subsection{Les principaux enjeux pour les réseaux de conservation}

Après avoir présenté l'organisation des réseaux de conservation de ressources génétiques au niveau national, nous allons identifier leurs contraintes et limites tant au niveau des firmes participant à la conservation qu'au niveau des relations entre les collections françaises et les réseaux internationaux (par exemple European Crop Plant Genetic Resources, ECP/GR, au niveau Européen).

\subsubsection{Au niveau des acteurs du réseau}

Quel que soit le type de conservatoire, l'intérêt de la conservation des ressources génétiques nécessite une valorisation économique : les graines ou germplasm conservés doivent être bien évalués de façon à avoir une valeur agronomique - face à un avenir incertain - liée aux caractéristiques principales (rendement, qualité, et résistance potentielle). L'intérêt des ressources génétiques dépend avant tout de l'utilité de leurs caractéristiques, ce que l'on retrouve dans la création variétale ${ }^{4}$ et l'amélioration végétale (impact économique non immédiat mais très fort) avec par exemple la création de pools génétiques dans les collections de maïs [13]. Ainsi, l'utilité n'est pas fondée

\footnotetext{
4 Sachant que l'amélioration végétale est une œuvre continue, il faut se placer sur une période assez longue pour apprécier le progrès génétique.
} 
sur la plante en tant qu'unité d'analyse, mais sur l'utilisation de ses caractéristiques (agronomiques, résistances, etc.) y compris la diversité génétique en elle-même, ce qui rejoint en économie les travaux de Lancaster [16]. Leur intérêt à long terme dépendra également des évolutions technologiques attendues avec la transgénèse et les organismes génétiquement modifiés [24].

Un point primordial à étudier, pour assurer la viabilité à long terme de la conservation, est lié au statut juridique des ressources génétiques. En effet, selon le système retenu, les possibilités d'appropriation des bénéfices liés aux innovations issues des collections nationales ou des collections de réseau seront différentes et pourront nuire à la conservation [25]. Cela engendre des options pour le statut des innovations qui seront issues de ce matériel (possibilité de protection ou non, de quels types, Certificats d'obtention végétale, $\mathrm{COV}$, ou brevets, de partage des bénéfices, etc.).

- Dans le cas d'une protection par COV (y compris dans sa version de 1991), système qui garantit la protection de la variété commercialisée et un libre accès aux ressources génétiques qui la composent, il semble qu'il y ait compatibilité avec la mise en œuvre d'une CN.

- Dans le cas du Brevet, l'analyse est plus complexe. Actuellement, la question porte sur l'interprétation des conditions de brevetabilité qui semblent s'être assouplies au cours de la dernière décennie, en particulier sur l'étendue réelle des brevets, seule la jurisprudence nous permettra de conclure sur cette question. Les brevets seront-ils accordés pour des usages identifiés ou pour tous les usages qui pourraient en être dérivés ? [21]. Il existe donc un risque de porter un coût d'arrêt à la conservation organisée en réseau, chacun ayant intérêt à conserver secrètes certaines recherches sur les caractéristiques et les possibilités de transférer celles-ci dans les variétés cultivées.

Parallèlement, il sera sans doute utile d'analyser les contrats de recherche qui lient les différents acteurs, en particulier au niveau du partage des droits de propriété intellectuelle [20]. En effet, ce n'est qu'à ce stade que l'on peut définir les incitations à la participation à un réseau de conservation. Surtout si les dernières phases de sélection et de création variétale sont prises en charge individuellement par chaque acteur.

Ainsi, selon le type de droit de propriété qui va s'imposer à l'avenir, il y aura un arbitrage entre type de protection intellectuelle / type de variabilité utilisée dans les programmes de sélection végétale, donc sur le type d'organisation de la recherche qui pourra favoriser l'émergence des $\mathrm{R} 1$ ou des $\mathrm{R} 2$. Cela risque de limiter le nombre d'échantillon mis en $\mathrm{CN}$ et/ou en collection de réseau, de même pour le niveau d'information diffusé.

\subsubsection{Au niveau international}

Les réseaux de conservation français, en raison de leurs collaborations internationales et nationales, sont au cœur d'une coordination internationale, de conservation des ressources génétiques. Par exemple, le Réseau Européen 
de céréales à Paille, dans le cadre des programmes ECP/GR, fait la même chose qu'en France mais à un niveau Européen. Le niveau central des projets ECP/GR collecte et coordonne de l'information sur le matériel, cette information provenant de centres de conservation ou de laboratoires de recherche qui, sur des préoccupations spécifiques, évaluent le matériel reçu, étudient les possibilités de croisements, effectuent les multiplications en vue des échanges et améliorent la variabilité génétique des souches utiles ou potentiellement utiles en sélection.

Le point clé au niveau international reste une question de définition de l'accès au matériel génétique et de définition de droits de propriété intellectuelle sur les innovations tirées des ressources conservées (négociés au niveau de l'Organisation mondiale du commerce, OMC). Au niveau international, les conditions d'échange de matériel ne sont pas encore fixées, la FAO proposant un système multilatéral d'échange alors que la CDB paraît favorable à un mécanisme bilatéral. Pour l'instant, les échantillons de la $\mathrm{CN}$ sont en libre accès pour les collections étrangères sous l'hypothèse d'une réciprocité des échanges mais sans contrats de type «Material Transfer Agreement» (MTA). Dans le futur, nous pouvons imaginer que le libre accès sera assuré pour les collections étrangères dont le pays aura signé l'accord multilatéral au niveau de la FAO ou, éventuellement, sur la possibilité de négocier des accords bilatéraux avec la France. Dans ce cas, participer à la CN permet d'élargir l'éventail des ressources génétiques étrangères accessibles, un sélectionneur recherchant un échantillon particulier proposera en échange l'ensemble des accessions de la $\mathrm{CN}$, et non plus des échantillons de sa propre collection.

\section{LES SÉLECTIONNEURS : DES ACTEURS DE LA CONSERVATION}

Dans l'analyse de l'organisation française de la conservation deux variables interviennent principalement : la coopération dans la conservation ; la coopération dans la recherche à des stades pré-compétitifs (création de pools géniques et recherche de fonctions). Ainsi, en passant de la collection de travail à l'adhésion à un réseau de conservation, les données du problème changent nettement pour les entreprises et différents avantages ressortent. Le matériel végétal est mieux évalué car le coût est partagé entre les membres du réseau. Il s'agit en premier lieu du matériel des organismes publics de recherche et du matériel cédé par les sélectionneurs.

\subsection{Organisation de la conservation, information et $R \& D$}

L'intérêt de la conservation en réseau est principalement d'ordre économique et social : l'analyse coût-bénéfice montre des résultats plus favorables lorsque le 
Tableau I. Statut du matériel végétal et information disponible.

\begin{tabular}{lccc}
\hline $\begin{array}{c}\text { Matériel } \\
\text { Information }\end{array}$ & Privé & Réseau & Public \\
\hline Privée & oui & Oui & oui \\
\hline Réseau & non & Oui & oui \\
\hline Publique & non & Non & oui \\
\hline
\end{tabular}

Source : Trommetter [25].

travail est réalisé en commun. Le fait que les recherches soient à un stade précompétitif est important : au-delà, les entreprises ne peuvent plus se différencier les unes des autres par leurs recherches personnelles, diminuant ainsi les chances de dégager des bénéfices substantiels par la suite.

Dans ce contexte, il est important de faire une distinction entre l'accès au matériel végétal et l'accès à l'information. Les membres du réseau ont libre accès à toute l'information sur les accessions de la collection de réseau. Par contre, les acteurs externes n'ont accès qu'aux données de passeport, voire aux caractérisations primaires, qui caractérisent le matériel végétal dans le catalogue de la CN. L'information supplémentaire concernant certains caractères d'intérêt agronomique peut être gardée secrète au sein du réseau et donc constituer des incitations à la participation.

Comme nous l'avons montré dans le tableau I, le niveau d'accès à l'information sur le matériel est inférieur ou égal au niveau d'accès au matériel. On peut avoir un accès public à du matériel sur lequel il existe une information génétique dont l'accès est limité aux acteurs du réseau voire à un acteur individuel, alors qu'il est impossible d'avoir de l'information génétique en accès public sur du matériel privé. Cela nécessite de définir des règles pour l'évaluation génétique et son financement. Si l'on recherche un caractère particulier (résistance ou qualité) :

- soit on évalue toute la collection sur ce caractère, sans bénéficier d'information génétique préalable ;

- soit on évalue collectivement une sous collection voire une core collection, puis on affine sur les populations de la collection qui sont, génétiquement, proches des échantillons qui se sont révélés les plus prometteurs.

La participation au réseau a un coût et présente certaines irréversibilités en terme d'engagement qui dépendent du type de réseau constitué (R1 ou R2). Par contre, elle permet d'avoir accès à un système multilatéral d'échange (si possible FAO, mais au minimum Européen, réseau ECP/GR).

La non-participation est sans coût, mais il existe deux incertitudes, la possibilité d'accéder au réseau international de façon bilatérale (sous quelles conditions de coûts ou de participation en nature) ainsi qu'une incertitude sur la 


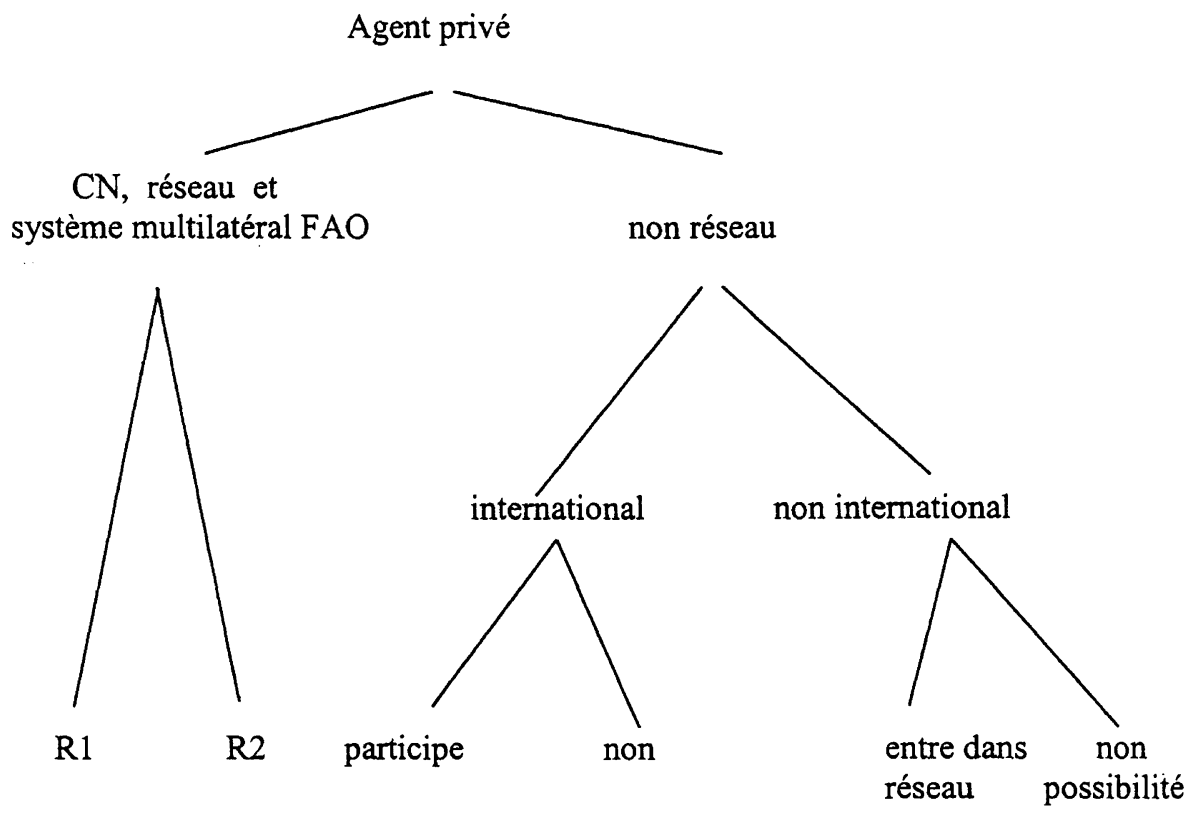

Figure 1. Modèle de comportement des acteurs.

possibilité d'intégrer le réseau $(\mathrm{R} 1, \mathrm{R} 2)$ au cours du temps et à quels frais ${ }^{5}$. Ces arbitrages de comportement pour les firmes sont résumés dans la figure 1.

\subsection{Cadre théorique mobilisé}

Notre objectif est d'identifier les variables qui interviennent dans la prise de décision pour adhérer à un réseau de conservation de ressources génétiques qu'il soit de type R1 ou R2 : accès à une plus grande gamme de ressources (probabilité de succès), meilleure connaissance du matériel végétal par évaluation (gain de temps de recherche) ce qui facilite les recherches dont les résultats sont la production d'objets d'intérêt collectif (stade pré-compétitif). Les principales contraintes sont liées aux coûts de l'engagement. Un certain nombre de questions ont émergé au cours de notre étude sur les réseaux de conservation. Elles portent généralement sur l'analyse de l'organisation du réseau : organisation de la conservation, organisation de la recherche et des relations qui existent entre les deux. Il s'agit donc d'assurer une conservation viable par l'utilisation des collections dans des programmes de $R \& D$ ce qui tisse le lien entre conservation et $\mathrm{R} \& \mathrm{D}$.

\footnotetext{
${ }^{5}$ Ainsi le coût d'accès au réseau peut dans certain cas être équivalent au coût cumulé individuel des adhérents précédents.
} 


\subsubsection{Organisation de la conservation : une question d'incitation}

Dans le cadre de l'organisme coordonateur de la conservation et de la gestion des ressources génétiques en France, l'objectif prioritaire est la conservation des ressources génétiques et la gestion de leur variabilité génétique. À ce niveau, en information parfaite, une question serait liée à l'étude et à la définition de l'équité dans le partage des tâches liées à la conservation et à l'évaluation de la $\mathrm{CN}$, question non résolue dans l'organisation actuelle avec nos deux types de réseaux ${ }^{6}$. Il existe, en effet, une information asymétrique entre le coordonateur et les différents acteurs du réseau, ce qui fait référence à la théorie des contrats et plus particulièrement au modèle Principal/Agents (Encadré 1). Ce cadre d'analyse part d'une situation en asymétrie d'information sur l'effort effectivement réalisé par les agents (en terme de conservation, régénération et caractérisation), dans lequel l'objectif est de définir des règles, voire des mécanismes institutionnels qui incitent les agents à réaliser des actions qui soient collectivement optimales [4].

\section{Encadré 1. Définition d'une relation principal/agent}

L'organisation la plus simple que l'on puisse imaginer est sans doute celle qui met en jeu deux parties, un employeur et un ouvrier, un propriétaire terrien et un fermier, un avocat et son client, une compagnie d'assurance et son assuré, un manager d'entreprise et l'État ou l'assemblée des actionnaires etc. Souvent l'une des parties, le mandant ou principal délègue au mandataire ou agent une action qui influence son bien être représenté par la production pour un employeur, la récolte pour le propriétaire terrien, la nature du jugement pour le client d'un avocat, etc. Si l'action de l'agent est observable, le Principal peut contrôler parfaitement l'agent sous réserve qu'il ait le pouvoir d'appliquer les pénalités nécessaires pour punir tout comportement déviant de l'agent.

En général toutefois, l'action de l'agent (niveau d'effort par exemple) n'est qu'imparfaitement observable. En effet, le résultat observé par le Principal est le produit joint d'une action que seul connaît l'agent et d'un aléa (que l'agent connaît ou ne connaît pas au moment ou il décide de son action). Le produit réalisé pour le compte du Principal par l'agent est donc aléatoire ; il dépend positivement d'une variable d'action de l'agent, non observable par le Principal et coûteuse pour l'agent. Le problème essentiel de cette mini-organisation est de définir des règles de partage du produit.

Source : Laffont [15], page 189.

${ }^{6}$ L'objectif de cet article n'étant pas de s'étendre sur la théorie de l'équité (pour une vision plus complète [6]), nous nous contenterons de signaler les plus courants : l'équité démocratique (Coût/Chiffres d'affaires équivalents), l'équité utilitariste (égalisation des coûts marginaux), etc., dont l'application aura des conséquences pour les acteurs qui ne seront pas nécessairement équivalentes, d'où la nécessité d'une décentralisation de la définition des cahiers des charges au niveau du réseau. 
L'incitation, pour l'agent, repose sur un partage des avantages et plus particulièrement des résultats qui peuvent découler des différentes étapes de la conservation, ce qui revient à une situation proche du partage d'un résultat collectif, développé par Alchian et Demsetz [2]. L'objectif de ces modèles étant d'éliminer les comportements de passager clandestin. De tels comportements peuvent exister dans la conservation des ressources génétiques du fait de l'existence d'un certain flou sur les conditions de participation au réseau.

Dans la charte, il est stipulé que chaque acteur choisit les échantillons qu'il met dans le réseau (ainsi que leur nombre), le partage des coûts et des tâches devant se faire de manière concertée (coûts de la conservation, coûts de contrôles, coûts de transaction, coûts de coordination, etc.). La définition du partage des tâches fait l'objet de négociations dans chaque réseau au niveau de la rédaction du règlement intérieur.

La desutilité pour les acteurs du réseau est donc principalement liée aux coûts de conservation et d'évaluation du matériel (desutilité croissante), et à la part de financement public dans ces opérations (desutilité décroissante). L'incitation est, quant à elle, liée à l'utilité que retirera une firme de sa participation à un réseau, utilité qui dépendra du matériel utilisé provenant de la collection ou des collections internationales, donc de ses activités de R\&D.

\subsubsection{Incitations : réseau de conservation et coopération dans la $R \& D$}

Il existe des difficultés pour inciter les firmes privées (acteurs du réseau) à participer à un réseau, sachant que les options institutionnelles ne sont pas encore établies et qu'il existe une incertitude sur les modalités de protection des innovations selon le type de matériel utilisé en sélection [25]. Il paraît clair que le mécanisme retenu va influer sur le nombre et le type d'accessions que les acteurs privés vont mettre dans la CN. Parallèlement, les questions informationnelles (type d'information, accès et transfert des connaissances, etc.) et de leurs conséquences sur la participation à un réseau de conservation ou non, seront au cSSur de l'analyse. On a, en effet, trois types d'information dans l'approche réseau : l'information publique sur le matériel génétique maintenu en banque ou en réseau (principalement données de passeport), une information privée qui est liée à l'évaluation individuelle du matériel par une firme et une information que nous qualifions de «collective » avec une réduction des délais de recherche par une meilleure information sur le matériel (comme nous l'avons vu dans le tableau I).

Ces deux groupes de questions (droits de propriété, accès à l'information, etc.) conduisent à une littérature liée à l'application de la théorie des contrats complets ou incomplets aux activités de R\&D. Pour les résoudre, on peut également utiliser une approche Principal/Agent (voir encadré 1). 
Ainsi, dans une recherche coopérative, s'il existe une situation de hasard moral - les actions des partenaires ne peuvent pas être toutes contrôlées (par exemple sur l'évaluation de caractères et l'incertitude sur la diffusion de l'information à l'ensemble du réseau) -, on peut identifier les conditions de succès et les limites de la coopération $[9,10]$.

Dans le cadre d'une relation Principal / agent, Aghion et Tirole [1] ont montré qu'en l'absence d'une bonne identification de la nature des innovations, il est possible de trouver une répartition optimale des droits de propriété entre les acteurs. En prolongement et sur la base d'une relation Principal - Multiagents, on peut chercher à construire un mécanisme incitant les entreprises à se regrouper (parallèle avec la création d'associations professionnelles) pour produire un effort collectif de recherche qui soit socialement efficace [22].

Dans notre problématique d'organisation de la $R \& D$ dans le secteur des semences, il semble que nous soyons plus proche des travaux qui portent d'une part sur l'étude d'un arbitrage entre multiplier les projets ou partager les coûts sur un projet dans le cas d'une recherche coopérative et d'autre part sur le rôle du partage de l'information dans la coopération en $R \& D[11,12]$. Dans ce dernier cas, il faut définir des règles de partage de la recherche et de la définition de droits d'usages de la nouvelle technologie, ce qui revient à renoncer aux gains de monopoles en cas de succès de la recherche. S'il y a partage des coûts et des résultats, la démarche sera en deux étapes : dans une première étape on a le choix de participer ou non à une recherche commune (arbitrage entre participer et ne pas participer), alors que dans une seconde étape, il faut fixer les règles de partage, les niveaux d'efforts de recherche de chacun, etc. La conclusion générale de ces travaux, est que plus la concurrence en aval est forte, plus un accord de coopération peut conduire à réduire l'effort global. Une telle difficulté semble être résolue (du moins en partie) avec des modèles basés sur des accords partiels de coopération [5] qui proposent une démarche en quatre étapes : recherche individuelle en investissant une somme fixe en $R \& D$ qui permet d'obtenir une information scientifique ; chaque firme dévoile tout ou partie de son information privée ; une course à l'innovation ; une concurrence.

\subsection{Le comportement des acteurs du réseau}

Par rapport aux développements théoriques présentés, notre objectif est de définir les caractéristiques de la conservation en réseau qui soient compatibles avec une participation des firmes dans le réseau. En effet, chaque firme a la possibilité de participer ou non à un réseau de conservation, selon les caractéristiques qui font que, pour une firme donnée, la participation au réseau est préférable à la non participation. Cela revient à définir les étapes de l'évaluation qui seront réalisées collectivement par les acteurs et donc à des logiques plus proches de celles de Combs $[10,11]$ ou de Bhattacharia et al. [5]. Notre approche peut se résumer ainsi : il existe une recherche coopérative avec : d'une part un accès libre aux ressources (sous couvert de MTA pour les non-adhérents au 
Tableau II. Les intérêts des entreprises à participer au réseau.

\begin{tabular}{lll}
\hline \multicolumn{1}{c}{ Intérêt économique } & \multicolumn{1}{c}{ Intérêt stratégique } \\
\hline $\begin{array}{l}\text { 1) Meilleure connaissance du } \\
\text { matériel végétal. }\end{array}$ & $\begin{array}{l}\text { 1) Veille sur l'évolution des rè- } \\
\text { gles internationales, voire volonté } \\
\text { d'infléchir cette évolution en mon- } \\
\text { trant une position française. }\end{array}$ \\
$\begin{array}{ll}\text { 2) Accès à un matériel végétal plus } \\
\text { large (dans le cadre national et }\end{array}$ & $\begin{array}{l}\text { 2) Rester au même niveau que les } \\
\text { international). }\end{array}$ \\
$\begin{array}{ll}\text { 3) Développer de nouveaux pools, } \\
\text { de nouveaux outils, de nou- }\end{array}$ \\
$\begin{array}{ll}\text { velles méthodologies (stade pré- } \\
\text { compétitif). }\end{array}$
\end{tabular}

réseau) mais pas à l'information stratégique (collective et privée) ; et d'autre part une incertitude sur le type de protection intellectuelle pour les innovations issues de matériel en libre accès.

\subsubsection{Les hypothèses générales}

La participation d'une entreprise à un réseau de conservation va dépendre d'au moins deux critères de décision : l'intérêt économique et l'intérêt stratégique qui sont résumés dans le tableau II.

Nous n'oublions pas qu'une partie non négligeable de l'effort de conservation et d'évaluation est réalisée par des stations de recherche publique. Mais nous nous intéressons uniquement aux conditions de participation des entreprises privées aux réseaux de conservation. Lorsque les membres sont des entreprises, l'intérêt va dans le sens d'une amélioration du profit à plus ou moins long terme. Rappelons que le matériel cédé par les entreprises aux collections de réseau n'est pas stratégique. Cela signifie donc qu'une analyse coût-bénéfice sur un horizon de 10-15 ans montrerait que les chances de créer un nouveau produit à partir de cette collection sont trop faibles pour engager des recherches individuelles.

\subsubsection{Les caractéristiques de l'adhésion}

L'objectif de cette présentation est d'essayer d'identifier les variables qui interviennent dans la décision d'une firme de participer à un réseau de conservation ou de négocier directement avec un grand centre de conservation. La question principale de cette recherche est : existe-t-il un gain lié à la participation à un réseau de conservation de ressources génétiques par rapport à la non participation (approvisionnement en ressources auprès de banques centra lisées) ? 
Par rapport aux modèles théoriques présentés dans la section précédente, nous allons donc analyser trois types d'organisation alternatifs :

- le premier est la non participation à un réseau avec un programme de R\&D classique dans lequel les firmes vont chercher, si possible (voir section 2.1.) du matériel dans les banques de semences étrangères (publiques ou privées) ou internationales dans une relation bilatérale ;

- le second où l'on participe à un réseau $\mathrm{R} 1$, mais où cette participation s'inscrit dans des programmes de R\&D classiques dans lequel les firmes vont chercher du matériel dans les banques de semences étrangères ou internationales en échange d'un accès à la CN constituée ;

- le troisième repose sur une organisation de type R2, avec une coopération en $\mathrm{R} \& \mathrm{D}$ à l'intérieur même du réseau.

Quelle que soit l'option retenue, les caractéristiques de l'organisation seront à trois niveaux :

- coopération au niveau de la conservation et de la régénération ;

- coopération au niveau de l'évaluation (caractérisation et évaluation) du matériel et du partage de l'information publique et stratégique entre les membres du réseau (caractéristiques potentiellement présentes dans un échantillon) ;

- recherche individuelle et droits de propriété intellectuelle sur les innovations.

L'analyse du comportement des firmes dans leur choix de participation à un réseau de conservation repose donc sur trois options qui seront analysées sous trois groupes de caractéristiques :

- de fonctionnement : conservation régénération et évaluation ;

- de recherche et d'innovations basées sur des probabilités de succès de la recherche ;

- d'options sur les droits de propriété intellectuelle, sur la mise en place, en France au niveau des CN, de MTA et sur l'évolution du statut des collections et des financements publics ${ }^{7}$.

Ces différents arbitrages sont présentés dans le tableau III où les avantages et inconvénients selon les configurations de réseau sont présentés en relatif (en ligne, entre les trois options de participation) en équivalent certains monétaire [15] :

$-(--)$ signifie très coûteux pour la firme et $(-)$ signifie coûteux ;

- (0) indifférent ;

$-(+)$ assez favorable, $(++)$ favorable alors que $(+++)$ signifie très favorable.

Dans le cadre du réseau de type $\mathrm{R} 1$, nous pouvons noter que la flexibilité de la participation et la recherche collective limitée conduisent à avoir généralement très peu de différence entre le nombre d'échantillons dans les collections de réseau et les $\mathrm{CN}$. En effet, l'objectif de la participation au réseau est principalement lié aux échanges de matériels avec les collections étrangères. Dans ce

\footnotetext{
7 Ainsi, dans le cadre de la charte nationale pour la gestion des ressources génétiques (signée par plusieurs ministères et organismes publics de recherche, [8]) la mise en œuvre d'un projet de classement des collections, d'accès au matériel (etc.) est avancé, ce qui correspond aux hypothèses présentées dans ce paragraphe.
} 
Tableau III. Caractéristiques des différentes options de participation.

\begin{tabular}{|c|c|c|c|c|}
\hline $\begin{array}{ll} & \text { Firme } \\
\text { Caractéristique } & \end{array}$ & \multicolumn{2}{|c|}{ Non réseau } & $\mathrm{R} 1$ & $\mathrm{R} 2$ \\
\hline \multicolumn{5}{|l|}{$\begin{array}{l}\text { Caractéristique } \\
\text { Fonctionnement }\end{array}$} \\
\hline \multicolumn{5}{|l|}{ Conservation et } \\
\hline régénération & \multicolumn{2}{|l|}{$\mathbf{0}$} & - & -- \\
\hline Évaluation & \multicolumn{2}{|l|}{$\mathbf{0}$} & - & -- \\
\hline \multicolumn{5}{|l|}{ Évaluation } \\
\hline Pools & \multicolumn{2}{|l|}{$\mathbf{0}$} & $\mathbf{0}$ & - \\
\hline Fonctions & \multicolumn{2}{|c|}{--} & - & - \\
\hline $\begin{array}{l}\text { Recherche et } \\
\text { probabilité de succès }\end{array}$ & $\begin{array}{l}\text { Accès banques } \\
\text { Internationales }\end{array}$ & Pas Accès & & \\
\hline Diversité & 0 & 0 & + & +++ \\
\hline Fonctions & ++ & + & +++ & +++ \\
\hline \multicolumn{5}{|l|}{ Options futures } \\
\hline Brevet & $+\mathrm{ou}-$ & + & + ou - & - \\
\hline MTA France & 0 & 0 & + & + \\
\hline $\begin{array}{l}\text { Statut et finance- } \\
\text { ment additionnels }\end{array}$ & 0 & 0 & + & ++ \\
\hline
\end{tabular}

contexte, même si la recherche de diversité est importante, elle sera beaucoup moins stratégique que dans les réseaux de type $\mathrm{R} 2$.

Dans le cadre des réseaux de type $\mathrm{R} 2$, la différence entre les échantillons de la collection de réseau et de la $\mathrm{CN}$ peut être significative, car les risques de pertes (d'appropriation par des acteurs externes au réseau) de caractères importants existent par l'absence de signature de MTA actuellement. L'organisation de la recherche est basée sur des recherches pré-compétitives sur des pools géniques (nouvelles lignées) et sur la recherche de fonctions.

L'absence de participation à un réseau sera grandement fonction des anticipations d'accès aux collections internationales. En effet, plus ces anticipations seront fortes et plus la non participation pourra se substituer à la participation à des réseaux R1 qui visent avant tout l'accès à du matériel étranger.

Cette présentation générale met bien en évidence les arbitrages entre ces différentes options, arbitrage qui dépendront à la fois des caractéristiques internes aux réseaux et des options institutionnelles nationales et internationales (ligne option dans le tableau III).

\subsubsection{Quels arbitrages}

Nous allons présenter deux arbitrages qui sont centraux dans le choix sur les options de participation à un réseau de conservation des ressources génétiques : 
l'arbitrage entre la non participation et l'adhésion à un réseau de type 1 ; l'arbitrage entre réseau de type $\mathrm{R} 1$ et de type $\mathrm{R} 2$.

\subsubsection{Participer ou non à un réseau}

L'arbitrage entre la non participation et la participation à un réseau $\mathrm{R} 1$ va dépendre du rapport entre les coûts de participation et la quantité de matériel, et/ou d'information, à laquelle on aura accès, donc à la probabilité de succès de la mise en œuvre d'un programme de recherche. Dans ce cadre, les entreprises ayant leurs propres stratégies de R\&D et d'alliances (entreprises hétérogènes), vont avoir des niveaux de participation différents (niveau d'effort au sens du modèle principal/agent) au réseau R1 en fonction de leurs caractéristiques propres (chiffre d'affaires, dépenses de recherche et développement, etc.) du fait de la flexibilité du règlement intérieur. Même si une telle situation, en situation d'information pure et parfaite, est théoriquement rationnelle et économiquement efficace, il existe des risques de comportements opportunistes pouvant conduire à une sous efficacité de la $\mathrm{CN}$ et de la collection de réseau, en terme de matériel et d'information collectée. Dans ce contexte, deux résultats pour le réseau $\mathrm{R} 1$ :

- l'introduction de droits de propriété intellectuelle de type brevet, au niveau international voire national, ne devrait pas avoir de conséquences trop irréversibles sur ces réseaux, du fait de l'organisation plutôt individuelle de la recherche et de l'accès aux $\mathrm{CN}$;

- l'introduction de MTA, pour l'accès aux collections françaises, et d'une reconnaissance institutionnelle forte (avec des fonds publics additionnels) devrait, par contre, permettre d'inciter à mettre plus de matériel dans les collections, donc d'augmenter les chances d'utilisation des collections et les probabilités de réaliser des innovations, du fait de la réduction du risque d'appropriation par des acteurs externes au réseau de caractéristiques intéressantes.

La participation à un réseau $\mathrm{R} 1$ est donc une fonction négative des possibilités d'accès individuellement à des collections de ressources génétiques et des coûts de la participation, par contre c'est une fonction positive de la mise en place de règles d'accès (MTA) et d'évolution du statut des collections. L'instauration d'un droit de propriété fort de type brevet, aura un impact négatif sur la participation, mais ne sera déterminant que dans des cas où les coûts de participation seront élevés.

\subsubsection{Quel type organisationnel de réseau choisir?}

L'adoption d'un réseau $\mathrm{R} 2$ par rapport à un réseau $\mathrm{R} 1$ dépend avant tout de l'organisation de la sélection pour l'espèce concernée. En effet, la création de pools géniques de réseau et la recherche de nouvelles lignées collectives, aux acteurs du réseau, nécessite la mise en place de règles en terme de partage des coûts et des résultats collectifs - accès à l'information immédiat 
ou différé pour les membres du réseau qui ne participeraient pas à certaines recherches collectives - qui visent à limiter les comportements de passager clandestin dans l'appropriation des recherches collectives (comme présenté dans la section théorique 3.2.) et peuvent donc conduire à des règles du type de celles énoncées dans l'exemple des R2 (dans la section 2.2.2).

Dans les R2, la CN mais aussi la collection du réseau est intéressante pour le matériel et surtout l'information qu'elle contient, les phases de recherches coopératives étant importantes, alors que dans le cas des R1, la CN sert principalement dans une configuration « échange » de matériel. Dans ce contexte, les options institutionnelles, retenues au niveau français et au niveau international, auront un impact non neutre sur le type d'organisation dominant :

- L'introduction des MTA et d'une reconnaissance institutionnelle forte aura un impact positif sur la CN avec l'introduction de nouvelles entrées pour les deux types de réseaux ;

- Par contre, l'introduction d'une protection des innovations par brevet, ou tout autre système sui generis qui limiterait l'accès aux ressources génétiques, pourra avoir des effets contre-incitatifs, surtout s'ils sont mis en place au niveau national. Cet effet sera fort pour la création de pools, du fait de la nécessité d'identifier les apports de chaque variété dans le pool final, pour assurer le versement de royalties au détenteur initial de la ressource [25].

Dans cet arbitrage entre types de réseaux de conservation, nous voyons qu' a priori plus les droits de propriété sur les innovations seront forts et plus les incitations à créer des réseaux de types $\mathrm{R} 1$ par rapport aux réseaux de type $\mathrm{R} 2$ seront importants. Néanmoins ce jugement doit être relativisé par l'existence d'outils - co-brevets ou licences obligatoires à condition d'accès facilité - qui, s'ils étaient adoptés dans le règlement intérieur du réseau, devraient être bénéfique pour la recherche coopérative des réseaux R2 (hors création de pools).

\section{CONCLUSIONS}

Dans cet article, nous avons présenté trois options pour la participation à des réseaux de conservation : la non participation, la participation flexible $\mathrm{R} 1$ et la participation égalitaire R2. Nous avons également présenté pour chaque option ses caractéristiques spécifiques en terme de coût de conservation et d'évaluation, et de probabilité de succès d'un programme d'amélioration végétale. Enfin, nous avons précisé dans la littérature économique les différentes questions à aborder, en particulier la question des incitations et du lien entre incitation à la participation à un réseau de conservation et organisation de la recherche dans les différents secteurs.

L'analyse de l'arbitrage entre les trois options d'adhésion à un réseau montre que dans le contexte actuel, il existe une différence significative entre l'organisation de la recherche dans les réseaux $\mathrm{R} 1$ et les réseaux $\mathrm{R} 2$ et que 
les réseaux de type $\mathrm{R} 1$ seront préférés à la non participation tant que la participation restera flexible. Dans le cas des réseaux $\mathrm{R} 2$, par contre, du fait d'un engagement plus important, il faudra que la probabilité de succès (sur les fonctions mais aussi dans la création de nouvelles lignées par la diversité) soit suffisamment forte et que les règles de partage des avantages, information et résultats collectifs, soient clairement établies.

L'analyse de la pérennisation de ces réseaux, en intégrant les anticipations sur les évolutions tant dans les droits de propriété que dans les institutions, montre qu'il peut y avoir des conséquences non neutres pour la conservation. Ainsi, nous pouvons noter que l'introduction de MTA au niveau des collections françaises devrait avoir une influence positive sur le matériel mis en CN, tant dans les collections de type R1 que R2, ce phénomène étant encore amplifié par la reconnaissance institutionnelle plus forte de ces CN. Par contre l'introduction du Brevet, ou de tout autre système sui generis contraignant, même s'il est relativement neutre quant au matériel conservé, il aura un effet plutôt négatif sur l'organisation des recherches collectives dans les réseaux $\mathrm{R} 2$ et donc risque d'entraîner une redéfinition des règles à l'intérieur de ces réseaux avec à terme un possible passage de R2 à R1.

\section{RÉFÉRENCES}

[1] Aghion P., Tirole J., The management of innovation, Quart. J. Econom., 109 (1994) 1185-1209.

[2] Alchian A.A., Demsetz H., Production, information costs and economic organisation, Am. Econom. Rev. (1972) 777-795.

[3] Amigues J.P., Desaigues B., A cost benefit analysis of biodiversity conservation programs in the Garonne valley, OCDE, 1999, X pages.

[4] d'Aspremont C., Gérard Varet L.A., Mécanismes de choix collectifs et incitations individuelles, Rev. Econom. Polit. 105 (1995) 657-679.

[5] Bhattacharya S., Sappington G.J., Licensing and the Sharing of Knowledge in Research Joint Ventures, J. Econom. Theor. (1992).

[6] Blanchard O., Criqui P., Trommetter M., Viguier L., Au-delà de Kyoto : enjeux d'équité et d'efficacité dans la négociation sur le changement climatique, à paraître in Econom. Prévis. 2000, 33 pages.

[7] BRG., Collection nationale France : Catalogue des ressources génétiques, blé tendre et orge. Réseau Céréales à paille, BRG ed., Paris, 1997.

[8] BRG. Charte Nationale pour la gestion des ressources génétiques, BRG éd., Paris, 1999, 99 pages.

[9] Choi J.P., Cooperative R\&D with moral hazard, Econom. Lett. 39 (1992) 485-491.

[10] Choi J.P., Cooperative R\&D with product market competition, Intern. J. Ind. Organ. 11 (1993) 553-571.

[11] Combs K.L., Cost sharing vs multiple research projects in cooperative R\&D, Econom. Lett. 39 (1992) 353-357. 
[12] Combs K.L., The role of information sharing in cooperative research and development, Intern. J. Ind. Organ. 11 (1993) 535-551.

[13] Gallais A., Monod J.P., La gestion des ressources génétiques du Maïs en France : de leur caractérisation jusqu'aux premiers stades de leur valorisation, C.R. Acad. Agric. Fr. 84 (1998) 173-181.

[14] Koo B., Wright B.D., The effects of advances in biotechnology on the optimality of ex-ante evaluation of genebanks materials, ICABR conference, Université de Rome, 1999, 38 pages.

[15] Laffont J.J., Cours de théorie microéconomique, Economica, 1991, 350 pages.

[16] Lancaster K.J, Socially optimal product differenciation, Am. Econom. Rev. $\mathrm{n}^{\circ} 4$ (1975) 567-85.

[17] Lefort M., Sontot A., Mitteau M., Chauvet M., Thaler L., La préservation des ressources phyto-génétiques : stratégie nationale et enjeux. CR Acad. Agric. Fr. 84, 1998, 183-194.

[18] Lefort M., Arbez M., Chauvet M., Dattée Y., Guiard J., Mitteau A., The french strategy for the management of plant genetic resources. Plant Variet. and Seeds 10, 1997, 153-162.

[19] Lemarié S., Recherche publique et dynamique industrielle : une illustration à partir d'un modèle évolutionniste appliqué au secteur des semences, Cah. Econom. Sociol. Rural. $\mathrm{n}^{\circ} 37$ (1995) 115-148.

[20] Lemarié S., Trommetter M., Quels dispositifs organisationnels faut-il mettre en place pour conserver et exploiter les ressources génétiques végétales ?, Journée théorie des contrats, IDEI/INRA, Toulouse, 1999, 25 pages.

[21] Noiville C., Ressources génétiques et droit : essai sur les régimes juridiques des ressources génétiques marines, Edition Pedone, 1997, 481 pages.

[22] Picard P., Rey P., Incentives in cooperative R\&D, in essays in Honor of E. Malinvaud, Camb. Univ. Press, 1991, 252-279.

[23] Salhuana W., Smith S., Maize breeding and genetic resources, International Symposium: Economics of valuation and conservation of genetic resources for agriculture, Université de Rome Tor Vergata, 1996, 18 pages.

[24] Trommetter M., Acteurs, droits de propriété et recherche dans le secteur végétal : une perspective historique, à paraître in Econ. et Soc., $1^{\mathrm{er}}$ trimestre, 2001, 20 pages.

[25] Trommetter M., Gérer la conservation des ressources génétiques : valeur et valorisation des collections, à paraître in Cah. Agric. 2000, $\mathrm{n}^{\circ} 9,381-389$.

[26] Trommetter M., How to evaluate a collection of Plant Genetic Resources? working paper 9706, INRA/SERD, Grenoble, 1997, 26 pages. 